\title{
Investigación-Acción Participativa en el Grupo denominado "Colectribu" de Jóvenes Artistas en situación de Riesgo Social
}

\author{
Participatory Action Research in the Group \\ "Colectribu" of Young Artists in Social Risk
}

\section{Pesquisa-ação participativa no Grupo: "Colectribu" de Jovens Artistas em Situação de Risco Social}

\author{
Daniel Augusto Padilla Bueno ${ }^{1}$ \\ https://orcid.org/0000-0002-8274-8586
}

Hugo Fernando Mamani Gutiérrez https://orcid.org/0000-0002-1486-1544

Ingrid Nicole Rioja Flores https://orcid.org/0000-0002-0243-1248

Carlos Federico Romero Flores \& https://orcid.org/0000-0002-9395-5353

Laura Patricia Trujillo Chávez https://orcid.org/0000-0003-2139-9631

1 Daniel Augusto Padilla Bueno es estudiante de la Carrera de Psicología de la Universidad Mayor de San Andrés en La Paz, Bolivia. Candidato a Máster en Resolución de Conflictos y Mediación. Correo Electrónico: dpadillabueno@gmail.com

- Hugo Fernando Mamani Gutiérrez es estudiante de la Carrera de Psicología de la Universidad Mayor de San Andrés en La Paz, Bolivia. Correo Electrónico: hugo.fernando.7@outlook.es Ingrid Nicole Rioja Flores es estudiante de la Carrera de Psicología de la Universidad Mayor de San Andrés en La Paz, Bolivia. Correo Electrónico: ingrid.nicole.rioja.flores@gmail.com Carlos Federico Romero Flores es estudiante de la Carrera de Psicología de la Universidad Mayor de San Andrés en La Paz, Bolivia. Correo Electrónico: carlosromeroflores@outlook.com Laura Patricia Trujillo Chávez es estudiante de la Carrera de Psicología de la Universidad Mayor de San Andrés en La Paz, Bolivia. Correo Electrónico: cucutrujillo@gmail.com 
https://doi.org/10.53287/blyk2648pi36c

Fecha de Ingreso: 20 de diciembre de 2020

Fecha de aprobación: 7 de mayo de 2021

Conflictos de intereses: Los autores declaran no tener conflictos de interés

\title{
RESUMEN
}

La presente investigación intenta describir la dinámica grupal desenvuelta entre miembros del grupo artístico de hip hop "Colectribu", conformado por jóvenes en situación de riesgo social, expuestos a fenómenos como violencia, pandillas, y consumo de substancias que asisten al programa "Resistencia Juvenil" dependiente de la Fundación La Paz. Se describe un espacio de desenvolvimiento artístico permitiendo el crecimiento de habilidades artísticas mediante talleres de lírica, producción musical y otros. Se plantea a través de esto la reformulación de la pandilla dejando atrás la intención violenta y de transgresión a las normas para conducir el trabajo de "Colectribu" hacia la creación, contribución a la sociedad y fortalecimiento personal. "[Colectribu] es la unión de jóvenes provenientes de distintas partes de La Paz que se reúnen en base a los que es el hip hop, es una forma de expresar a las personas un mensaje nuevo, consciente y reflexivo". Se realiza un estudio de acción-participativa que además de ser plasmado en dicha investigación, se realiza un documental catalogando la realidad de los miembros del grupo que puede ser encontrado en https://youtu.be/fiX6eR1FV4A.

\section{PALABRAS CLAVE}

Psicología de grupos; Pandillas; Jóvenes; Hip Hop; Investigación-Acción Participativa.

\begin{abstract}
This research tries to describe the group dynamic that unravels between members of the hip hop artistic collective "Colectribu", consisting of young people in social risk, exposed to phenomena such as violence, gangs and substance abuse, they attend the "Resistencia Juvenil" program dependent from the La Paz Foundation. It describes a space of artistic development allowing growth of artistic abilities by means of lyricism workshops, musical production and others types of workshops. At the same time the concept of gang reformulates, leaving behind the violent intent to redirect the work from "Colectribu" to creation, social contribution and personal growth. "[Colectribu] is a union of youth who come from different places of La Paz who get together joined by hip hop, it's a way of expressing to people a new message, that is conscious and reflexive". An action-participative study was made, as well as a documentary who logs the reality of the members of the group that can be found at https://youtu.be/fiX6eR1FV4A.
\end{abstract}

\section{KEY WORDS}

Group psychology; Gangs; Youth; Hip Hop; Participatory Action Research.

\section{RESUMO}

Esta pesquisa procura descrever a dinâmica de grupo desenvolvida entre os membros do grupo de arte hip hop "Colectribu", formado por jovens em situação de risco 
social, expostos a fenômenos como violência, gangues e uso de substâncias que participam do programa "Resistência Jovem" dependente da Fundação La Paz. É descrito um espaço de desenvolvimento artístico que permite o crescimento de habilidades artísticas por meio de oficinas de letras, produção musical e outras. Com isso, propõe-se a reformulação da quadrilha, deixando para trás a violenta intenção e violação das normas de conduzir o trabalho de "Colectribu" à criação, contribuição à sociedade e fortalecimento pessoal. "[Colectribu] é a união de jovens de diferentes partes de La Paz que se reúnem com base no que é o hip hop, é uma maneira de expressar uma mensagem nova, consciente e atenciosa para as pessoas". É realizado um estudo de ação participativa que, além de refletido na referida pesquisa, é realizado um documentário catalogando a realidade dos membros do grupo que pode ser encontrada em https://youtu.be/fiX6eR1FV4A.

\section{PALAVRAS-CHAVE}

Psicologia de grupo; Gangues; Juventude; Hip Hop; Pesquisa-ação participativa.

\section{Introducción}

El proyecto "Investigación e Interacción Social en el Grupo Denominado "Colectribu" perteneciente a la Iniciativa "Resistencia Juvenil" plantea un modelo que, siguiendo la teoría propuesta por Lewin (1946, citado en Torres, 2016) que es necesario estudiar a un grupo en la totalidad de sus dimensiones, no a los individuos por sí solos o desde un exterior, a la vez de que los investigadores deben inmiscuirse en las actividades y vivencias del grupo; se utilizó la técnica de la observación participante.

Se muestra la realidad del grupo desde un análisis teórico y práctico en áreas tales como caracterización del grupo, motivación, comunicación y liderazgo.

Como objetivo se plantea describir y categorizar al grupo "Colectribu" como parte de la iniciativa "Resistencia Juvenil de la Fundación La Paz", quienes buscan empoderar a jóvenes en situación de riesgo, creando resiliencia y alejarlos de medios peligrosos. Al mismo tiempo se plantea la descripción de fenómenos grupales tales como tipo de grupo, motivación, comunicación y liderazgo. Obtener lecturas y vi- vencias participando de las actividades espontáneas de las que los miembros forman parte, sean batallas de gallos, juntadas de torneos de baile, talleres, etc. Plasmar las vivencias y lectura de la realidad del grupo a través de medios audiovisuales.

Se nos ha permitido empaparnos de la realidad que transita "Colectribu", como investigadores ofrecimos una descripción desde diferentes aspectos grupales en la espera de que sea de utilidad para tratar distintas problemáticas en esta iniciativa social o en similares en el contexto de la ciudad de La Paz, para devolver este conocimiento a la población y, por último, empoderarlos.

\section{Método}

El enfoque se da desde la investigación-acción participativa, método de investigación psicosocial basada en la inmersión e interacción de investigadores y participantes de una comunidad en sus propias prácticas, cultura, costumbres, y su reflexión sobre sí mismos (Guzmán, 2020). Cualquier conocimiento generado durante el proceso investigativo ha sido usado para el beneficio de la comunidad meta (Lewin \& Salazar, 1992). 


\subsection{Instrumentos}

Para llegar a este conocimiento se hace el uso de las técnicas de observación participante, en la cual el investigador interactúa socialmente con los sujetos observados, se influyen mutuamente en el desarrollo de su realidad de manera holística (Ruíz, 2020). Se hace también el uso de guías de entrevista, siendo una conversación con propósito investigativo, recopilando información de una forma completa y objetiva en un ambiente de respeto, prudencia y cordialidad, cada entrevista podrá sufrir modificaciones en función de la información recabada y el interés de ahondar en diferentes tópicos (IBERTIC n.d.).

\subsection{Participantes}

El procedimiento es realizado con 5 miembros activos y 5 pasivos del grupo "Colectribu", jóvenes de entre 18 a 24 años de edad, grupo anidado dentro de la Fundación La Paz cuyo equipo de trabajo incluye 4 administrativos en cargos de director de culturas, director de proyecto, psicóloga y otros.

\subsection{Procedimientos}

En la fase de acercamiento, se consensua con los líderes del equipo que los investigadores serán incluidos a las actividades cotidianas del grupo "Colectribu", esto incluye acompañarlos a cualquier taller, concierto, salida informal o formal que tengan programada. El equipo acompaña y recoge sus percepciones a través de grabaciones audiovisuales a clases de baile, ensayos, batallas de gallos, festivales de bandas, conciertos públicos, sesiones de organización grupal, y otras actividades más. Una vez generado el vínculo e identificado a los miembros más influyentes del grupo, se procede a realizar entrevistas bajo una guía de preguntas, que buscan entender la historia, la tendencia de liderazgo y las características desarrolladas en los resultados. Todos estos momentos son grabados en audio y video con el pleno consentimiento de los miembros del grupo. Una vez recabada la información es clasificada en categorías que responden a liderazgo, motivación, comunicación, etc.

\section{Resultados}

\subsection{Categorización de grupo}

Los jóvenes que participan de esta iniciativa se definen a sí mismos como: "[Colectribu de villa]; es la unión de jóvenes provenientes de distintas partes de La Paz (ciudad donde se ubica el proyecto) que se reúnen en la Fundación La Paz y crean una visión, una misión y un proyecto a base del hip hop, que es una forma de expresar a las personas: "nosotros somos artistas que queremos dar un mensaje nuevo, consciente y reflexivo".

Con la intención formal que manifiestan al querer cumplir sus objetivos como artistas, posteriormente se han formado lazos afectivos, amistades y compañerismo dentro del grupo, es así que llegan a denominarse como una familia, dándole un carácter de grupo informal, estos grupos surgen sin una designación oficial otorgada por la organización en la que se alojan, sino que son espontáneos y se basan en relaciones interpersonales o intereses en común (Schermerhorn, 2004). Coincidiendo con las afirmaciones del director del proyecto, quien manifiesta que la intención del programa no fue formar un grupo como "Colectribu", si no que los mismos jóvenes decidieron usar el Hip Hop y crear el colectivo de rap por iniciativa propia, mientras que la organización apoyó esta idea.

El lazo es reforzado con la convivencia diaria que se forma dentro de la fundación, deja de ser regida por responsabilidades o tareas hacia "Resistencia Juvenil" o sus programas, compartiendo actividades tales como ir a la plaza Camacho de la ciudad de La Paz a interactuar con otros grupos, o a presentaciones de cada uno 
de los miembros que no sean organizadas por "Resistencia Juvenil", pasando a caracterizarse como un grupo primario, quienes moldean el yo social de la persona, proporcionando motivos, normas y valores que guían su conducta y su autoimagen (Gonzáles, 2009).

Como manifiestan los miembros, en el grupo comparten la concepción de la realidad, experiencias y vivencias aprendiendo unos de los otros, no solo en técnica de rap o de producción, sino también discuten sus vidas personales y reciben consejos unos de otros, convirtiéndolos a su vez en un grupo de referencia, dando como consecuencia la asimilación de los sentimientos del grupo y referir su conducta a los valores del grupo con el fenómeno de conformidad (Merton, 2002, citado en Abreu, 2012) También muestran rechazo en la interacción con otros grupos, que, a pesar de ser de pares, y tener en común hábitos en los que se involucraban (consumo de sustancias o crimen) los empujan a la búsqueda de otras relaciones con personas que no repitan estos comportamientos, que tengan valores y normas como los de Colectribu.

\subsection{Motivación}

La motivación en el grupo es intrínseca, persiguiendo metas por el disfrute de hacerlo, a la vez la conformación del grupo se da para satisfacer necesidades de validación y reconocimiento social que genera un sentido de responsabilidad con ellos mismos para alcanzar un reconocimiento positivo de la sociedad dentro del rol de artistas; los componentes de "Colectribu" afirman que entre ellos existe una autoexigencia para mejorar las expectativas artísticas del grupo de unos en comparación con otros.

Festinger (1954, citado en Psychology Notes $H Q, 2017)$ menciona que los motivos serán sociales en función de que conlle- ven una conducta que implique la interacción con otras personas; es así que en la comparación social evaluamos nuestras habilidades en comparación de otros; se evalúan las habilidades y destrezas propias con sujetos que poseen habilidades parecidas.

"Colectribu" suple las siguientes necesidades como motivación:

- Motivación al logro y de crecimiento siendo definida por McClelland (1989) como el impulso a sobresalir, alcanzar las metas, esforzarse para tener éxito, buscar hacer algo mejor, es entonces que los miembros del grupo manifiestan: "para que un joven no, no tenga que estar perdiendo su tiempo en la calle, con los amigos, las drogas y el alcohol, más si es que le gusta esto de la música que se venga aquí a la fundación, acá lo vamos a apoyar, ayudar y hacer crecer y que se enfoque para que realmente logre algo en esta vida y que a sus 30 años no se esté lamentando de no haber hecho lo que le gusta"

- Necesidades de afiliación y fisiológicas son atendidas (Maslow, 1943 citado en García-Allen, 2015) "con el Colectribu que congenio tanto, veo tanto y hablamos tanto, nos influenciamos tanto y convivimos de esta manera yo ya los veo como mi familia porque si ellos necesitan comida pues vamos les doy comida, si ellos tienen sed tomemos agua, si ellos quieren hablar hablemos, yo soy como un hermano para ellos...es bonito tener amigos que se vuelvan hermanos" "aquí tengo apoyo psicológico.... amistad, compañerismo, siempre te escuchan, si alguna vez me ven raro me preguntar qué ha pasado, siempre me preguntan si he comido, me invitan en lo posible". 
- $\quad$ Motivación al logro (McClelland, 1989) de personas que prefieren colaborar antes que competir, además de necesidades de auto realización, ya que según ellos: " [se busca] hacer un antes y después en el hip hop boliviano. hacer algo bueno por esta sociedad más que todo". "Dar un mensaje y que esto crezca, un colectivo de raperos que quieran hacer música y se apoyen entre ellos, crear y no competir"

- Necesidad de reconocimiento (Maslow, 1943 citado en García-Allen, 2015) se hace presente y atendida, manifestada así por los participantes: "la búsqueda de apoyo como bases del grupo... está en dos dimensiones, la primera tiene que ver directamente en cómo se relacionan entre ellos... cada uno forma parte, coopera a su manera...el hecho de encontrarse entre pares pero con un objetivo en común... la segunda dimensión es de la fundación, no solo les damos un espacio físico sino también colaboramos mucho con espacios que no son físicos... de reconocimiento por parte de todos, de ellos entre ellos como personas independientemente de género, de ellos como sujetos de derechos, de pares... reconozcan a sí mismos como personas, individuos, sujetos de derecho, grupo autónomo que tiene derecho a tomar decisiones" la sociedad rechaza a la comunidad hip hop por prejuicios, el equipo de trabajo no se ve limitado por los mismos desarrollando una relación horizontal con los miembros del grupo.

- Se promueve la autorrealización a partir de la fundación como organismo incubador "si bien la fundación actúa como una casa o un lugar donde se los acoge, también ellos en algún momento tienen que ser independientes y tienen que poder surgir más allá de que la fundación siga o no, es importante darle al grupo o instrumentos para sostenerse.... maneras de relacionamiento, con círculos restaurativos por ejemplo ellos se han ido dando cuenta de cómo podría funcionar una dinámica de relación en la que se pueda hablar los problemas o resolver las diferencias...que las van a tener como cualquier grupo humano"

Para entender las verdaderas necesidades del grupo, fue necesario desarrollar una relación próxima, así lo manifiestan los ejecutivos del proyecto Resistencia Juvenil "se han abierto diferentes talleres en función a los interés y necesidades de los jóvenes, pero a veces si tenemos un lazo no muy fortalecido a veces simplemente responden con algo que se les aparece en la cabeza..."quiero clases de guitarra" se genera el espacio pero llegan la primera sesión, la segunda, ya para la tercera o cuarta no continúan asistiendo... a partir de generar un lazo de confianza entre personas del mismo nivel, más allá de la institución o de lo que yo haga... a partir de ese lazo se ha escuchado las verdaderas necesidades que ellos tienen, como fortalecer esto que implica las batallas de gallos....ya no quedarse en la modalidad de calle, salvaje, con baratas, saben que impactan pero violentándolo... eran mejor reconocidas si conocían contenidos históricos o culturales sin malas palabras"

Los jóvenes dependientes de la fundación imitarán las maneras de trato por parte del equipo a manera de comparación social, evaluando sus propias habilidades, características y actitudes al otro parecido (Festinger, 1954 citado en Cherry, 2020) "mantenemos una relación horizontal y esto va a ser efecto de lo que los chicos 
ven allá en la oficina, si nosotros como equipo nos mantenemos en una relación horizontal y de respeto los chicos van a notar eso y a reconocerlo".

\subsection{Comunicación}

La comunicación en "Colectribu" y la fundación La Paz provee una estructura al contenido lírico que plasman y comunican a través de sus canciones, "aprendimos métrica, estructura, tipos de poesía...son como llaves o formas de hacer algo que nos ayudan, por ejemplo los cuartetos nos ayudan en el rap porque se divide en cuatro tiempos y nos ayuda full al escribir y también al flow porque dependiendo a cuantas sílabas le pongas a la línea en donde estas rapeando en un cuarteto es muy esencial y es en donde tu flow sobresale".

El grupo Colectribu entiende comunicación como una actividad terapéutica "hablamos de cosas personales, es como una buena terapia porque a veces uno necesita contar las cosas personales para un poco liberarse y todo aquello ¿no? Entonces compartimos cosas personales, anécdotas, cosas graciosas a veces también puedes contar si no te está yendo bien en algo y te sientes apoyado". Esta comunicación no se da en subgrupos o dúos, es extendida hacia todos los miembros participantes "no estamos de parejitas y no hablar con otros, nos movemos y hablamos entre todos, es fluido".

Además de ello, se marca una diferencia entre lo que es comunicación y socialización, caracterizando a la primera como formal y limitada, la segunda como más cercana y afectiva en sentido de convivencia "con los b-boys, con los MCs, con los DJs, con los grafiteros siempre ha habido una comunicación, siempre un hola, buenas tardes y chau. Acá en Colectribu ya fue más socialización, empezamos a conocernos y poco a poco anduvimos por las mismas calles, durmiendo en la misma casa, comiendo de la misma comida". Ba- sados en la retroalimentación, convivencia y emoción.

Dentro de la colaboración artística, encontramos signos de comunicación asertiva (Da Dalt de Mangione \& Difabio de Anglat, 2002), siendo la misma la capacidad de expresar sentimientos, ideas, opiniones y creencias de manera efectiva y directa sin agresividad "el tallerista estaba ayudando a personas a como grabar y nosotros contribuimos. "bro añadile esta palabrita va a sonar mejor... a veces se molestaban cuando les ayudábamos, eso depende, pero la idea es que si les decimos también está bien porque sabes que va a sonar mejor y hay otros que es más difícil el ayudar porque el cuate es muy malo y él quiere rapear y el gana, pero se puede explicar con más calma y puede decir "haber intentaré" y lo hacen en ese momento y se da cuenta de que funciona mejor. Hay problemas cuando él no quiere recibir nuestros comentarios, pero se respeta", al compartir información se realiza a manera de sugerencia y con respeto al otro.

El fenómeno de la pelea de gallos tiene una característica de agresión verbal (AKANA, 2018), definido como un enfrentamiento buscando dominar "es la oportunidad para decirte a la cara todo lo que no te diría de frente" "puedes decir cualquier cosa pero que no pase a puños", sin embargo, existen ciertos criterios para que un verso en la batalla de gallos sea más valorado "lo que se toma en cuenta ya lo que se da más validez es a la inteligencia, es decir a las frases o las palabras que están bien usadas, el ingenio ... se tienen que fijar en el flow (la manera en la que las palabras acompañan la música), como fluye en el beat (el ritmo de la música)".

Se considera talento en las batallas de gallos a: "alguien que hable rimado sobre un beat, que intercambie ideas, porque el que te dice insultos sin lógica del porque te lo ha dicho no es un buen competidor". Esto tiene un similar al criterio de comuni- 
cación de dos vías necesaria para una a compresión, se observa también que los versos muchas veces son construidos en base a los que el otro acababa de decir en el anterior.

Colectribu es caracterizado por el uso de círculos restaurativos "alguien agarra un objeto y es el que habla, los demás no pueden dar su opinión hasta que le pasen el objeto y nos empezamos a explayar... vamos conociéndonos entre nosotros y a nosotros mismos por dentro... hace crecer al unión y la hace más fuerte", que definida por ellos mismos será "desarrollar los conflictos, y en la mejor situación lo arreglamos y todos bien, que ambos lados queden satisfechos" es utilizado para la resolución de los conflictos entre sus miembros "Dejarlo hablar al que quiera hablar, dándole la palabra, escuchándolo y pues, nos generamos desde adentro, está prohibido de que alguien se burle porque puede que alguien haya tenido el valor de hablar y de contar algo está prohibido burlarse. Nos basamos mucho en la empatía también, de siempre llevarnos bien y ser sinceros, sin filtros, puedo decirle que puede cambiar su actitud si puede hacerlo bien y si no también o no sé porque se comporta así, habría que saber porque se comporta así no?...Pero también puede que uno de los dos no quiera entender y también ahí vemos los demás nuestro punto de vista en el caso que las decisiones que estén tomando afecten al grupo porque también toman decisiones que son más propias y uno respeta la decisión de los demás" se intenta restaurar el vínculo dañado entre los miembros participantes, para posteriormente reparar el daño causado, si no se llega a un acuerdo el grupo interviene con diferentes puntos de vista del problema, es importante resaltar que este proceso ocurre sin la intervención de personas ajenas al grupo, y las sesiones son dirigidas por los miembros más antiguos o activos de los mismos.
Estas prácticas previenen, gestionan y resuelven el conflicto dentro de los grupos, reforzando los vínculos afectivos entre los afectados, mediante preguntas reparadoras, diálogos sobre el problema y las soluciones (Campus FAD, 2013).

Debido al uso de los mismos por parte del grupo estudiado consideramos pertinente su definición. Los círculos restaurativos son una adaptación de prácticas indígenas de todo el mundo, que enfatizan la reparación y aprendizaje a través de un proceso grupal y colectivo opuesto al castigo. Son experiencias enmarcadas en la filosofía de la Justicia Restaurativa y rescatan creencias aborígenes sobre la necesidad de que las personas estén en buena relación con su entorno. En los círculos participan todas las partes involucradas en un conflicto de modo pacífico y no confrontacional, para hablar del problema y buscar una solución. Si bien el proceso puede incluir la restitución del daño causado, está diseñado en primera instancia para sanar o recomponer las relaciones entre personas dentro de la comunidad, antes que para imponer un castigo (Lorente, s.f.).

Consideran que el hip hop es una herramienta artística de solución de conflictos a través de la comunicación "tenemos obviamente conflictos pero gracias al hip hop todo se soluciona hablando, o con una batalla de rap, o con una batalla de break dance y ahí sabemos que quizás él me ha ganado porque el practica más, el mete más empeño, yo digo que tengo que meterle más empeño, pero no se hace para subir el ego, sino más bien para bajar el ego y subir la humildad, si él me ha ganado merece respeto... todo se soluciona hablando".

Además de la resolución de conflictos, la actividad de círculos restaurativos puede tener diferentes finalidades, abarcando la reflexión sobre temas que incitan a compartir experiencias personales, autoconocimiento y conocimiento de los miembros del 
grupo "puede ser una especie de actividad donde haciendo ciertas preguntas sobre una temática podemos analizar y reflexionar todos, porque a veces uno se sincera $y$ quiérase o no pueden salir cosas personales, y por eso pueden solucionarse problemas personales, no necesariamente tiene que haber un conflicto entre dos personas... es una terapia que ayuda a que una persona pueda superar ciertos conflictos".

\subsection{Liderazgo}

La iniciativa "Resistencia Juvenil" nace como un intento de formación de líderes, al mismo tiempo se obtiene una descripción de las características de líder que ellos conciben: "Antes esto era como un lugar para formar líderes, gente que aprenda a tomar el mando que sepa sobre valores, (transcender, responsabilidad, etc.) conductas, (enseñar o tutorear, su experiencia) o incluso leyes para poderse defender en la calle, saber nuestros derechos y sobre todo aprender a tener un lenguaje asertivo (hablar de forma correcta ante las personas $y$ ante las situaciones)... te ayuda mucho a nosotros siendo raperos siendo gente que lo que decimos queremos que sea escuchado".

Los líderes identificados por los miembros de "Colectribu", tienen un estilo de liderazgo democrático mayormente, involucrando a los miembros del grupo en la toma de decisiones y utilizando la retroalimentación como medida para corregir errores, facilitando la integración (Fierro \& Villalba, 2017). A su vez se encuentran con rasgos autoritarios y laissez-faire como minoritarios (Aiteco Consultores, 2019). Los líderes son reconocidos por la información que poseen, su habilidad técnica, y su edad "Chicho es el productor, nos enseña y nos tutorea y eso lo hace un líder quizás" "A un principio yo lo veía a Álan como... él es más pro que nosotros y nos está enseñando por eso, entonces lo he visto como un líder en algún punto hasta que el mismo nos dice que somos un colectivo y que nadie es más que ninguno o sea todos comparten sus ideas y creamos entre nosotros, yo soy igual que ustedes y ustedes igual que yo, pero si lo admiramos, al tallerista de poesía y hay algunos también que tienen muy buen nivel y el mensaje que transmiten".

Las jerarquías son aplanadas en el intento de búsqueda de horizontalidad, resaltando la individualidad y a su vez en la igualdad. "líder es alguien a quien le hayamos dicho vas a ser el representante o el presidente o el máximo exponente, no, todos son artistas independientes en un grupo colectivo como una familia, pero nos apoyamos todos, ese es el propósito aunque seamos independientes" "Alguien quería decirnos que hacer y le decíamos estamos en la misma altura, bájate nomás digamos de la silla, nos sentaremos todos igual, tratar de decirnos lo más honesto posible lo que nos molesta" "Se crea una unión a través de hacer los que le gusta, donde no hay jerarquía y todos son iguales".

"Todos somos iguales, más lo único que cambia son nuestros nombres, pero todos somos humanos y aprendemos unos de otros... acá lo que nos une como Colectribu los que ya tienen algo más, como Chicho que nos enseña muchas cosas que en su momento se vuelve un jefe, después viene Maya que nos empieza a dar consejos y nos tutorea, y a veces yo que a todos los chicos les hablo y me escuchan, a veces Lios, somos un ayllu, un grupo que está creciendo junto y no tenemos jefes, no tenemos un tesorero o presidente".

Se entiende por Ayllu una unidad sistémica, configurada por sus mismos elementos y abarca todas las esferas de la vida social, comunidad (Qamasa, 2011).

A pesar de la horizontalidad es posible identificar a guías desde diferentes campos de acción. 
"Hemos podido aterrizar el tema de jerarquías, se reconocer que hay personas más proactivas podemos decir, que expresar mejor sus ideas o son participativas, está muy bien y se va a reconocer eso, pero tratamos de mantener una relación horizontal aquí en el grupo".

"Los cabezas serían los más adultos, los que ya tienen experiencia en la movida, el Álan y el Chicho, los que más estamos activos también".

Es entonces que la antigüedad y la proactividad son vistas a su vez como indicadores de liderazgo.

\section{Discusión}

El grupo autoformado dentro de la iniciativa "Resistencia Juvenil", es un claro ejemplo que la investigación-acción participativa es una estrategia válida para generar planes de intervención y potenciamiento comunitario. El proyecto comienza con la intención de reunir a jóvenes con un propósito formal de formación de liderazgos, pero no puede limitarse a solo brindar estos servicios y espacios, ya que los lazos naturales que los miembros del grupo tienden a formar, además de su necesidad natural de expandirse y consolidarse con una identidad propia exigirán más de quienes intervinieron en un primer momento, los encargados del proyecto deben instaurar costumbres comunes que en un momento deben dejar de ser intervenidas por los mismos y absorbidas por el grupo, para que como meta final, el grupo sea autosostenible, y forme nuevos grupos similares al mismo, con las diferentes metas y necesidades que cubren.

Se comprende que las limitaciones de la IAP están sujetas al compromiso de los sujetos, el cual no llegó a los miembros pasivos de "Colectribu", además de la dificultad de incluir instrumentos estandari- zados por las mismas limitaciones epistemológicas y de vínculos que promueve la IAP, llegando a la posibilidad de lastimar la conexión natural que establece el investigador con los sujetos.

Se reconoce a su vez los conflictos internos que evidencia el grupo, es entonces que los mismos miembros deben trabajar con la ayuda de sus líderes para enfrentar estos asuntos de una forma constructiva, sin dejar que los elementos agresivos de su lenguaje ofusquen el progreso y las herramientas desarrolladas para una comunicación asertiva que ya han desarrollado.

Por último, una intervención completa del estilo IAP tiene una duración aproximada de 1 a 2 años, las limitaciones de tiempo nos han permitido mostrar la realidad del grupo a sí mismo y devolver el conocimiento para su propia construcción de estrategias y resolución de problemas, lastimosamente no seremos capaces de ver el cambio o estas estrategias desarrollarse de manera natural en los miembros del grupo, además de que la inclusión de los investigadores al ritmo de grupo de trabajo de los líderes pudo haber tenido consecuencias en la interrupción de sus labores o planes para su iniciativa.

\section{Conclusiones}

En un inicio "Colectribu" fue un grupo de referencia con una estructura formal, dependiente de la Fundación La Paz dentro del programa de "Resistencia Juvenil" que tiene como objetivo ayudar a los jóvenes en situación de riesgo y pandillas a conformar grupos que se enfoquen en la música y el baile. Con el paso de los años "Colectribu" se convierte en un grupo de pertenencia que mantiene estructura formal, pero a su vez ha generado relaciones interpersonales conformando grupos informales como respuesta a la necesidad de contacto fraterno e íntimo social. 
"Colectribu" es también un grupo primario, que alberga un número de miembros que comparten un sentimiento de pertenencia.

"Colectribu" es grupo que se polariza cuando hay dificultades para el cumplimiento del compromiso normativo, asumiendo decisiones creativas que toman en cuenta todas las opiniones y comprenden que tienen derecho a no estar de acuerdo y que estas interacciones mejoran con el paso del tiempo, la cohesión y comunicación en el grupo. Como grupo mantienen la motivación intrínseca por que persiguen metas por el disfrute de hacerlo, a la vez la conformación del grupo se da para satisfacer necesidades de validación y reconocimientos sociales como motivación extrínseca que genera un sentido de responsabilidad con ellos mismos para alcanzar reconocimiento positivo de la sociedad como artistas; los componentes de "Colectribu" afirman que entre ellos existe una autoexigencia para mejorar las expectativas artísticas del grupo de unos en comparación con otros.

En Colectribu se manifiestan comunicación no verbal que les ayuda a dirigir mensajes cuando no pueden expresarlo verbalmente en la pelea de gallos; también hacen interacción en sentido unidireccional, que utilizan para anunciar eventos en los que participaran utilizando redes sociales, otra es la comunicación de dos vías que generalmente es para resolver conflictos o para generar mayor cohesión. Los directivos y encargados del proyecto utilizan la comunicación asertiva mientras que los componentes del grupo comunican sus vivencias a través de la música de forma agresiva pasiva y asertiva en los círculos restaurativos. Utilizan al Hip Hop como instrumento de resolución de conflictos.

La fundación La Paz ha tratado de manejar de manera horizontal aplanando jerarquías, aun así, se ha identificado tipos de liderazgo: democrático como mayoría, rasgos autoritarios y laissez -Faire como minoría. El grupo identifica al liderazgo democrático como factor motivante para incrementar potencialidades artísticas. El grupo considera la antigüedad y la proactividad como indicadores de liderazgo.

El documental fruto de la presente investigación puede ser encontrado en https:// youtu.be/fiX6eR1FV4A

\section{Referencias bibliografícas}

Abreu C. (2012) La Teoría de los Grupos de Referencia. Universidad Nacional de

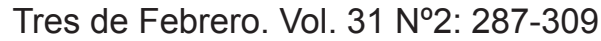

Aiteco Consultores. (2019) Kurt Lewin: Estilos de Liderazgo. https://www.aiteco. com/kurt-lewin-liderazgo/

Akana Psicología. (2018, 18 de junio). Los estilos de respuesta. https://www.akanapsicologia.com/los-estilos-de-respuesta/

Campus FAD. (2013, 21 de julio). Los CÍRCULOS RESTAURATIVOS, Una HERRAMIENTA más para la GESTIÓN de la CONVIVENCIA. https://www.campusfad. org/blog/accion-magistral/los-circulos-restaurativos-una-herramienta-mas-para-la-gestion-de-la-convivencia/

Cherry, K. (2020, 20 de septiembre). How social comparison theory influences our views on ourselves. Verywell Mind. https:// www.verywellmind.com/what-is-the-social-comparison-process-2795872

Da Dalt de Mangione, E. y Difabio de Anglat, H. (2002) Asertividad y su relación con los estilos educativos familiares. Interdisciplinaria: revista de psicología y ciencias afines. Vol. 19, № 2.

Fierro, I., \& Villalva, M. (2017). El liderazgo democrático: una aproximación conceptual. INNOVA Research Journal, 2(4), 155-162. https://doi.org/10.33890/innova. v2.n4.2017.210 
García-Allen, J. (2015, 26 de marzo). Pirámide de Maslow: La jerarquía de las necesidades humanas. Psicología y Mente. https://psicologiaymente.com/psicologia/ piramide-de-maslow

Guzmán, G. (2020). Investigación Acción Participativa: ¿qué es y cómo funciona? https://psicologiaymente.com/social/investigacion-accion-participativa

Gonzáles R. (2009) Diccionario Crítico de Ciencias Sociales. Terminología Científico-Social, Ed. Plaza y Valdés.

IBERTIC (s.f.) Entrevistas a profundidad, guías y pautas para su desarrollo, de https://oei.org.ar/ibertic/evaluacion/pdfs/ ibertic_guia_entrevistas.pdf

Lorente, R. (s.f.). Los círculos restaurativos, otra herramienta de convivencia. CAREI. https://carei.es/wp-content/uploads/Rosa-Lorente-Blog-circulos-restaurativos.pdf Lewin, K., \& Salazar, M. C. (1992). La investigación-acción participativa: Inicios $Y$ desarrollos.

McClelland, D. (1989). Estudio de la motivación humana. Narcea Ediciones.
Psychology Notes HQ (2017). Leon Festinger's Social Comparison Theory. https:// www.psychologynoteshq.com/leonfestinger-socialcomparisontheory/

Qamasa, O. (2011). Apuntes acerca del sistema de cargos en los Ayllus bolivianos._SCIELO._www.scielo.org. bo/scielo.php?script $=$ sci_arttext $\&$ pi$\mathrm{d}=\mathrm{S} 0040-29152011000100009 \& I n-$ $\mathrm{g}=$ es\&nrm=iso

Ruíz, L. (2020). Técnica de observación participante: tipos y características. https:// psicologiaymente.com/psicologia/tecnica-observacion-participante

Schermerhorn, J. (2004). Comportamiento organizacional. Editorial Limusa S.A. De C.V.

Torres, A. (2016, 6 de junio). Kurt Lewin y la Teoría del Campo: el nacimiento de la psicología social. Psicología y Mente. https://psicologiaymente.com/social/kurtlewin-teoria-del-campo 